\title{
Monte Carlo analysis of a control technique for a tunable white lighting system
}

\author{
Chakrabarti, Maumita; Thorseth, Anders; Jepsen, Jørgen; Corell, Dennis Dan; Dam-Hansen, Carsten
}

\section{Published in:}

Lighting Research and Technology

Link to article, DOI:

$10.1177 / 1477153517691332$

Publication date:

2017

Document Version

Peer reviewed version

Link back to DTU Orbit

Citation (APA):

Chakrabarti, M., Thorseth, A., Jepsen, J., Corell, D. D., \& Dam-Hansen, C. (2017). Monte Carlo analysis of a control technique for a tunable white lighting system. Lighting Research and Technology, 50(5), 716-728. https://doi.org/10.1177/1477153517691332

\section{General rights}

Copyright and moral rights for the publications made accessible in the public portal are retained by the authors and/or other copyright owners and it is a condition of accessing publications that users recognise and abide by the legal requirements associated with these rights.

- Users may download and print one copy of any publication from the public portal for the purpose of private study or research.

- You may not further distribute the material or use it for any profit-making activity or commercial gain

- You may freely distribute the URL identifying the publication in the public portal 
Monte Carlo analysis of a control technique for a tunable white lighting system

\author{
M Chakrabarti , A Thorseth , J Jepsen , D Dan-Corell and C Dam-Hansen \\ Department of Photonics Engineering, Technical University of Denmark, Lyngby, \\ Denmark
}

Short title: Monte Carlo analysis of control for tunable white lighting

Received 14 October 2016; Revised 30 December 2016; Accepted

A simulated colour control mechanism for a multi-coloured LED lighting system is presented. The system achieves adjustable and stable white light output and allows for system-to-system reproducibility after application of the control mechanism. The control unit works using a pre-calibrated lookup table for an experimentally realized system, with a calibrated tristimulus colour sensor. A Monte Carlo simulation is used to 
examine the system performance concerning the variation of luminous flux and chromaticity of the light output. The inputs to the Monte Carlo simulation, are variations of the LED peak wavelength, the LED rated luminous flux bin, the influence of the operating conditions, ambient temperature, driving current, and the spectral response of the colour sensor. The system performance is investigated by evaluating the outputs from the Monte Carlo simulation. The outputs show that the applied control system yields an uncertainty on the luminous flux of $2.5 \%$ within a $95 \%$ coverage interval which is a significant reduction from the $8 \%$ of the uncontrolled system. A corresponding uncertainty reduction in $\Delta u^{\prime} v^{\prime}$ is achieved from an average of 0.0193 to 0.00125 within $95 \%$ coverage range after using the control system.

Address for correspondence: Maumita Chakrabarti, Skibhusvej 133, 5000 Odense C, Denmark.

Email: maumita@hotmail.co.uk

\section{Introduction}

Mixed colour LED white lamps and luminaires are used widely in theatres, studio lighting, and architectural lighting due to their wide gamut, outstanding energy 
efficiency $^{1-3}$, and attractive colour quality ${ }^{4-6}$. These systems, based on additive LED colour mixing, present challenges regarding the stability of colour and light output during the operation and the lifetime of the systems. The instabilities are due to variations in colour and luminous flux of the individual LEDs that are large enough to be perceived by human observers. These types of changes in light output can be noticeable when comparing the illumination from similar lighting systems side by side. The stability and the reproducibility of such lighting systems depend on both the intrinsic properties of the LED system and the operating conditions. The intrinsic properties, i.e. the wavelength and the luminous flux of the LEDs within the selected bin, and the sensitivity of individual LEDs to the operating conditions, can be varied. The abrupt failure of LEDs can also cause lumen depreciation. Dong et al diagnosed lumen depreciation in multi-coloured LED systems ${ }^{7}$, mainly due to LED failures, using a system with photosensors which may limit the real world implementation because many such systems use a lot of LEDs. Finally, the operating conditions themselves can vary notably; e.g. current level, ambient temperature, junction temperature ${ }^{8}$, 
operation time, aging, etc. ${ }^{9}$.

To keep colour and light output variations within acceptable levels for critical applications, some form of active control of the LED system is necessary. Without compromising the efficiency and the light quality, several methods of controlling light have been implemented by other authors ${ }^{10-21}$. Temperature feedback alone can account only for the variations of the luminous efficiency with the temperature changes for the coloured LEDs. Thus, this feedback system is not in common practice. On the other hand, feedback of the luminous flux alone cannot interpret the variations with temperature of the chromaticity coordinates of the coloured LEDs. It can account for the variations of luminous efficiency with the aging of LEDs. Therefore, the combination of temperature feedback and luminous flux feedback are well suited for LED colour control. The chromaticity coordinates feedback system measures the chromaticity coordinates and at the same time as the brightness of the mixed light produced by the LED luminaire directly. This kind of feedback system is also appropriate for the LED colour control. Moreover, there is no strong preference for 
employing either the combination of temperature feedback and luminous flux feedback or feedback for chromaticity coordinates ${ }^{22}$. Application specific requirements decide the choice of the feedback system to obtain the quantitative evaluation of the feedback system. In this paper, we have controlled chromaticity coordinates along with the light output of a multicoloured LED lighting system which has an application in stage lighting. Furthermore, we have evaluated the uncertainty of the system parameters in real time by using a Monte Carlo simulation.

In this paper, we propose an approach where we utilize a set of initially optimized settings and a colour sensor to control the light output and chromaticity during the operation of a multi-coloured LED lighting system. By implementing a feedback loop based on the colour sensor readings, the control system delivers an error correction through the optimized settings for the individual colour channels to produce a tunable white light according to the target values of the correlated colour temperature (CCT) and the luminous flux $\left(\Phi_{V}\right)$. These target values could range in CCT from $3000 \mathrm{~K}$ to $6000 \mathrm{~K}$ with a dimming capability and in $\Phi_{V}$ from 1000 to $8000 \mathrm{~lm}$, thus 
achieving a minimum lower dimming limit of $13 \%$ for a particular CCT. Dimming below $1000 \mathrm{Im}$ for that particular CCT could introduce an uncertainty from the limitations of the LED driver electronics known as digital multiplex (DMX) control. Due to the uncertainties in the lighting system, the light output will deviate from the target of CCT and $\Phi_{V}$. The Monte Carlo simulation process is applied to calculate the uncertainty in the output of the LED lighting system from the influences of LED luminous flux and wavelength bin, junction temperature, driving current, ambient temperature, DMX control on current, and colour sensor response. The advantage of the Monte Carlo simulation is that there is no requisite for partial derivatives of the complicated equations or analytical propagation of uncertainty. The methodology applied here is a combination of simulated and experimental findings of the uncertainties of individual input parameters of the coloured LEDs. Although the present simulation shows the influence of luminous depreciation on colour stability, in future, further simulation can be done on the uncertainty contributions due to the unexpected failure of LEDs and LED ageing. The probability distribution of output chromaticity parameters $u^{\prime}, v^{\prime}$, CCT, 
$D_{u v}$, and $\Phi_{V}$ are used to evaluate the uncertainties of input parameters. The control unit uses an incorporated, pre-calibrated lookup table for an initial estimate and then evaluates the result by using a calibrated tri-colour sensor for monitoring the chromaticity of the light output. After that, the control unit utilizes the lookup table and the colour sensor output to set a new set of driving currents to the individual colour channels by choosing a new lookup entry. The stability of the chromaticity is quantified by using the CIE method for evaluating white light sources ${ }^{23}$ which uses the $\Delta u^{\prime} v^{\prime}$ distance in the CIE 1976 uniform chromaticity diagram.

\section{Methods}

We propose a new method that permits the regulation of colour and luminous flux while minimizing erroneous variations for a particular system using knowledge of a generic lighting system. The method incorporates a pre-calibrated lookup table that employs a rule-based optimization of chromaticity distance from the Planckian locus $\left(D_{u v}\right)$ for the original CCT and $\Phi_{V}$ settings. The calibrated colour sensor, monitoring the 
tristimulus values $(X, Y, Z)$ is applied to provide feedback from the light output. The Monte Carlo simulation is used to provide the statistical analysis of the uncertainties on the output parameters resulting from the uncertainties on the input parameters to the multicolored LED lighting system. A schematic diagram of the control system is shown in Figure 1. The whole control mechanism is divided into two sections: 1 ) the top-left is called the control algorithm; 2) the middle section is showing the hardware connections to the control unit. The right section of Figure 1 presents the statistical analysis of the input parameters and their corresponding influences to the control mechanism while the bottom-left evaluates the Monte Carlo output. The following sections explain the procedures of the control unit in detail.

\subsection{Pre-calibrated lookup table}

We initially mixed the spectral radiant flux of four coloured LEDs (royal blue, blue, red, and green) with a warm white LED. The additive colour mixing process supplements and changes the spectral power distribution (SPD) of the white LED. This multi-coloured light engine is described in Chakrabarti et $a l^{24}$. The spectral radiant flux of the coloured LED is calibrated from the spectral measurements of the individual coloured LED at various settings of the controllable parameters (operating current, junction temperature, etc. $)^{25}$. In Figure 2, we illustrate two resultant SPDs - shown with the black line, which are the outputs for two of the target CCTs $(3000 \mathrm{~K}$ and $5800 \mathrm{~K}$, respectively) at 8000 lumens. In Figure 2 , the purple line represents the reference 
SPDs, which for CCT $\leq 5000 \mathrm{~K}$ is represented by a Planckian radiator SPD and for CCT > $5000 \mathrm{~K}$ is represented by the corresponding D-illuminant SPD. The other coloured SPDs in Figure 2 show the contribution from individual colour channels to create the resultant SPD. The lookup table is set up with a novel algorithm. The Table uses the reference SPDs, to find the target CCTs ( $3000 \mathrm{~K}$ to $6000 \mathrm{~K}$ in $1 \%$ step size of the previous CCT $)$ and the corresponding target $\Phi_{V}(1000 \mathrm{Im}$ to $8000 \mathrm{Im}$ in $3 \%$ step size of the previous $\Phi_{v}$ ) with an optimized $D_{u v}$. The target colour settings for the light output are also optimized by the general colour rendering index (CRI) $R_{a}>85$ and the particular $\mathrm{CRI} R_{g}>40$ for rendering red objects. The target point in the lookup table offers information about the corresponding DMX values for the individual colour channels for the LED light engine as described in Chakrabarti et $a l^{24}$.

\subsection{Colour control}

To operate the LED lighting system, the user would have to set the target CCT to a value between ( $3000 \mathrm{~K}$ and $6000 \mathrm{~K}$ ) and the luminous flux $\Phi_{V}$ to a value between (1000 Im and $8000 \mathrm{Im}$ ) [Figure 1]. The control unit then finds a match in the lookup table closest to the target values. In the ideal case, the lighting system delivers the target by using the corresponding DMX values of individual colour channels from that lookup point. The aforementioned uncertainty contributors inside the lighting system influence the light output. Unlike the analytical analysis, the uncertainty analysis of multi-coloured LEDs can be done by the Monte Carlo simulation process with an 
iteration $N=2000^{26}$.

We use the Monte Carlo simulation to investigate the colorimetric and photometric variations of the spectral light output as output $\left(u^{\prime}, v^{\prime}\right.$, and corresponding CCT, $D_{u v}$, and $\left.\Phi_{V}\right)$ based on the uncertainties generated from the lighting system as input to the Monte Carlo simulation [Figure 1]. These input uncertainties are measured experimentally or are adopted from data sheet values. With this methodology, similar but varying lighting systems, utilizing e.g. other LED bins and environmental conditions, can be controlled without doing any additional calibrations. The control system explained in Aldrich et $a l^{21}$ did not consider the aforementioned uncertainty parameters inside their lighting system to produce the optimized lighting solution. In our paper the colour sensor has been used to monitor the CCT, $\Phi_{V}$, and $D_{u v}$ variations in real-time and receives the feedback from the light output (Figure 1). The intensity dimming of our system is quicker than the aforementioned system. The used colour sensor has approximate tristimulus responsivity of the colour matching functions $(\bar{x}, \bar{y}$, and $\bar{Z}$ ) and needs to be calibrated close to the operating chromaticity range to minimize the spectral mismatch errors. The colour sensor was calibrated by using a handheld spectrometer (GS-1150) ${ }^{27}$.

After obtaining the measured tristimulus values of the resulting white light, the control algorithm calculates the corresponding $\mathrm{CCT}_{\text {Measure, }} \Phi_{V \text { Measure, and }} D_{u v}$ Measure from the tristimulus values. After that, the $\mathrm{CCT}_{\text {Measure, }}$ and the $\Phi_{V \text { Measure }}$ are subtracted from 
the $\mathrm{CCT}_{\text {Target, }}$ and the $\Phi_{V \text { Target }}$, respectively. These differences are then added to the

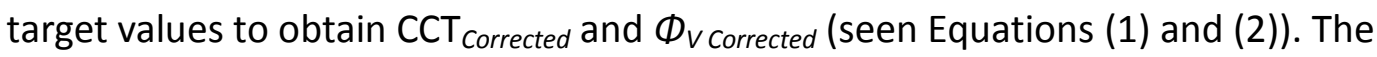
closest values of the CCT and the $\Phi_{V}$ to the $\mathrm{CCT}_{\text {corrected, }}$ and the $\Phi_{V \text { corrected }}$ are then found in the lookup table.

$$
\begin{gathered}
C C T_{\text {Corrected }}=C C T_{\text {Target }}+\left(C C T_{\text {Target }}-C C T_{\text {Measure }}\right) \\
\Phi_{V \text { corrected }}=\Phi_{V \text { Target }}+\left(\Phi_{V \text { Target }}-\Phi_{V \text { Mrasure }}\right)
\end{gathered}
$$

The new lookup point provides the corrected driving information for the individual colour channels to deliver a corrected light output, which is seen in the result section to be closer to the target CCT and $\Phi_{V}$ values. The lookup table already has the optimized $D_{u v}$ values $\left( \pm 2 \cdot 10^{-3}\right)$ for each lookup point. However, the large variation in $D_{u v}$ may need an extra $D_{u v}$ correction. In that case, the $D_{u v}$ needs to be corrected using the individual colour sensitivity to $D_{u v}$. If the obtained $D_{u v}$ shows a higher value than the desired $D_{u v}$ value, the green channel needs to decrease along with adding more from red and/or blue channels depending on the CCT region. For a negative $D_{u v}$ correction, the green channel needs to increase while the red and/or blue channel needs to drive less than the previous settings. The white channel can increase/decrease to adjust the $\Phi_{V}$. Finally, after the $D_{u v}$ correction, the changes in 
driving currents of the respective colour channels lead to a new lookup point, which is evaluated by plotting on the CIE $1976\left(u^{\prime}, v^{\prime}\right)$ diagram ${ }^{23}$. We cannot control colour rendering indices ${ }^{28}$ (such as $R_{a}, R_{9}$ ) by the proposed control technique. However, the pre-calibrated lookup table already has been spectrally optimized to $R_{a}>85$ and $R_{9}>40$. Thus, the controlled light output has a good light quality regarding colour rendering according to the application. The control mechanism can be generalized to be used in any other solid state lighting system where instead of using the colour sensor, a spectrometer may be employed for obtaining the spectral information.

\section{Results and discussion}

As mentioned earlier, there is a challenge in getting a stable light output from a multicoloured LED lighting system due to the different performances of the LEDs under the same operating conditions which are the inputs for the Monte Carlo simulation. Using the Monte Carlo method to simulate the variation of input parameters of individual LEDs internally for $N=2000$ iterations, the uncertainties in the colour quantities $\left(u^{\prime}, v^{\prime}\right.$, $\mathrm{CCT}, D_{u v}, \Phi_{V}, R_{a}$ and $R_{g}$ ) in light output are obtained as output from the Monte Carlo simulation. To achieve colour quantities, the Monte Carlo simulation results in 2000 simulated resultant SPDs for the 2000 iterations (acting as 2000 virtual lighting systems). Due to the uncertainties in the system, those 2000 SPDs are different to the 
target SPD, resulting in variations in the CCT, $\Phi_{V}$, and $D_{u v}$. The simulated light output for tuning range from $3500 \mathrm{~K}$ to $6000 \mathrm{~K}$ and shows an average of $8 \%$ change on $\Phi_{V}$ within a $95 \%$ coverage interval over an initial setting of $8000 \mathrm{Im}$ due to the uncertainties of the input parameters if the ambient temperature changes from $20^{\circ} \mathrm{C}$ to $45^{\circ} \mathrm{C}$.

We have taken $5500 \mathrm{~K}$ and $8000 \mathrm{Im}$ as target CCT and $\Phi_{V}$, respectively (as seen in Figure 1) to illustrate the colour variations in the uncontrolled multi-coloured LED lighting system. The Monte Carlo method was then applied to the target SPD to add changes due to uncertainty contributors for the 2000 iterations. From the resulting 2000 SPDs, the chromaticity coordinates $\left(u^{\prime}, v^{\prime}\right)$ are calculated. The probability distributions of variations in CCT and $D_{u v}$ for the 2000 iterations are derived from ( $u^{\prime}$, $\left.v^{\prime}\right)$ and are shown in Figure 3(a)-(b). The coverage interval of 95\% from the distribution implies $\Delta C C T=940 \mathrm{~K}$ with an arithmetic mean of $5709 \mathrm{~K}$ and $\Delta D_{u v}=0.0252$ with an arithmetic mean of 0.0028 , and are represented by the green colour in the same figure. In Figure 3(c) and (d) we have plotted the corresponding probability distributions of variations in CRI $R_{a}$ and $R_{g}$ derived from the SPD of the light output from the Monte Carlo analysis. Within the $95 \%$ coverage interval, indicated by green lines, we have found the CRI variation of $\Delta R_{a}=15.4$ with an arithmetic mean of 91 and a change of $\Delta R_{9}=95.7$ with an arithmetic mean of 43 , respectively. These high values of the colour rendering indices come from the spectral optimization algorithm, used for generating the pre-calibrated lookup table. Similarly, the coverage interval of $95 \% \Delta \Phi_{V}$ 
is equal to $704 \mathrm{~lm}$ with an arithmetic mean of $8170 \mathrm{Im}$. We have evaluated the uncontrolled output parameters of CCT, $D_{u v}$, and $\Phi_{V}$ from the Monte Carlo simulations for target CCT settings from $3500 \mathrm{~K}$ to $6000 \mathrm{~K}$ with a target $\Phi_{V}$ of $8000 \mathrm{~lm} . \Delta \mathrm{CCT}, \Delta D_{u v}$, $\Delta R_{a}, \Delta R_{9}$, and $\Delta \Phi_{V}$ are large for the uncontrolled lighting system [Figure 3]. Thus, the observer can perceive the colour variations as well as light output variations. However, these variations can be narrowed down within a certain limit after implementing the control mechanism as described in the Method section (Figure 1). Table 1 shows the variations in CCT, $D_{u v}$, and $\Phi_{V}$ for the uncontrolled lighting system compared with those of the controlled system. The Table shows six target CCT settings from $3500 \mathrm{~K}$ to $6000 \mathrm{~K}$ while all are at a target of $\Phi_{V}=8000 \mathrm{~lm}$. The uncontrolled $\Delta C C T, \Delta D_{u v}$, and $\Delta \Phi_{V}$ are high at a target CCT of $3500 \mathrm{~K}$. This is because, at a target CCT of $3500 \mathrm{~K}$, the contribution from the uncertainties due to the red LEDs are higher than for the other target CCTs. From the Table, it can be seen that the stabilized system decreases the average uncertainty within a $95 \%$ coverage interval on luminous flux from $8 \%$ to $2.5 \%$. The reduction in chromaticity from the uncontrolled system of average 0.0193 and to the controlled system is of average 0.00125 in $\Delta u^{\prime} v^{\prime}$ within $95 \%$ coverage range. Similarly, the controlled system reduces the uncertainty on the CCT at $5500 \mathrm{~K}$ from $940 \mathrm{~K}$ to 133 $\mathrm{K}$. The corresponding maximum $D_{u v}$ variation is reduced from 0.025 to 0.001 at $5500 \mathrm{~K}$. Similar values are found at the other target CCTs.

Figure 4 shows the distribution of the four parameters for the 2000 iterations 
after implementing the control system at a target CCT of $5500 \mathrm{~K}$. The green lines show the distribution within a 95\% coverage interval. Figure $4(a)$ and $(b)$ indicate that the variations of CCT and $D_{u v}$ have been reduced to $\Delta C C T=133 \mathrm{~K}$ and $\Delta D_{u v}=0.0011$ within the $95 \%$ coverage interval. Similarly, Figure $4(\mathrm{c})$ and (d) show $\Delta R_{a}=0.18$ and $\Delta R_{9}=4.9$ within $95 \%$ coverage range.

However, when the user insists on exceeding the delivery capability of the luminaire, the control system cannot provide solutions within the tolerance region of the target luminous flux. Table 2 demonstrates the simulated results of a target CCT at $3000 \mathrm{~K}$ while the target luminous fluxes are $4000 \mathrm{Im}$ and $8000 \mathrm{Im}$, respectively, for uncontrolled and controlled lighting systems. In that table, for the target value $4000 \mathrm{Im}$, the control system provides controlled $\Phi_{V}$ with $2.2 \%$ variations over the $95 \%$ coverage interval. However, for the target value $=8000 \mathrm{Im}$, the solutions of $\Phi_{V}$ lie within $5 \%$ variations over the $95 \%$ coverage range because all the red LEDs were not performing at the required level.

Table 3 shows the simulated results for the accepted luminous flux depreciation of each colour LED implemented in the present control system. The Table also indicates the maximum number of admitted LED failures during the controlling operation. The considered amount of LED failures among 210 LEDs is $4 \%$ in total. In future, to handle more abrupt failure and luminous flux depreciation, each LED needs to be operated at a maximum of $80 \%$ of its available power. 
Figure 5 indicates the effective performance of the control system by showing the colour variations of 2000 iterations in the CIE $1976\left(u^{\prime}, v^{\prime}\right)$ diagram before and after having the colour control applied to the lighting system. In Figure 5, the deviations in $D_{u v}\left(\Delta D_{u v}\right)$ are seen as the distance from a given point to the daylight locus (black solid line). The variations in CCT ( $\triangle C C T)$ are found as the distance to the isothermal line (black dash lines). A just noticeable chromaticity difference at $50 \%$ probability is considered to be 0.0013 in $\left(u^{\prime}, v^{\prime}\right)$ coordinates, corresponding to $n=1.1^{23}$. This definition is usable in the white operating region which is investigated here. Figure 5 shows a Just Noticeable Difference (JND) circle at $n=1.18$, and a circle with a radius of double that of the JND circle corresponding to $n=2.36$. Here the centre of the circle is the target $\mathrm{CCT}=5494 \mathrm{~K}$, and $D_{u v}=5.89 \cdot 10^{-4}$ (purple triangle).

It is clear that the target position is indicating an offset from the daylight locus. This offset is because the lookup values are not optimized to $D_{u v}$ at zero, rather; they are optimized to $D_{u v}$ at $\pm 2 \cdot 10^{-3}$ as recent findings show that the human preference for $D_{u v}$ favours an optimum $D_{u v}$ that might not be at zero $D_{u v}{ }^{29,30}$. From Figure 5 , it is observed that the found solutions before implementing the control system (gray dots) are almost outside the just noticeable chromaticity difference circle (solid red) and they are not closer to the target point. Similarly, the green 'o' implies the solutions for those 2000 iterations after implementing the control system in Figure 5. It is observed from Figure 5 that $95 \%$ of the controlled chromaticities are within a circle of $n=2.36$, 
hence the colour control system can narrow down the variations along the daylight locus $(\triangle C C T)$ as well as along the isothermal line $\left(\Delta D_{\text {uv }}\right)$. The system is not able to control all the chromaticities within the JND circle.

The present lookup table has two dimensions for ranges of target values in CCT and $\Phi_{V}$, where the targets represent an optimized $D_{u v}$ value $\left( \pm 2 \cdot 10^{-3}\right)$. Here we have used a separate $D_{u v}$ correction separate from the lookup table. Future work may improve the controlled solutions by implementing a three-dimensional lookup table by adding a range of $D_{u v}$ values.

Figure 6 describes the simulated results of the control system for the range of CCTs of the lighting system at $\Phi_{V}=8000 \mathrm{Im}$. The CCT of the lighting system is tunable from $3000 \mathrm{~K}$ to $6000 \mathrm{~K}$ with a step of $100 \mathrm{~K}$. The red solid 'dot' data points represent the controlled CCT point obtained as a solution at the corresponding target CCT. The relation between them is linear with a coefficient of determination $R^{2}=0.999$. The controlled luminous flux found at the corresponding target CCT is denoted by the blue solid "square" data points, while the dashed black line is showing the target: $\Phi_{V}=8000$ $\mathrm{Im}$. The variation in luminous flux at $8000 \mathrm{Im}$, for the CCT ranging from $3000 \mathrm{~K}$ to 6000 $\mathrm{K}$ is within $\sim 1.8 \%$. The control mechanism can be validated by experimental LED colour mixing systems with colour sensors installed to demonstrate both the system stability and the system-to-system reproducibility determined visually and by spectroradiometric measurements. 
Figure 7 shows the influence of lumen depreciation on $\Delta u^{\prime} v^{\prime}$ at a target CCT of $3500 \mathrm{~K}, 4500 \mathrm{~K}$, and $5500 \mathrm{~K}$ where the simulated luminous depreciation is employed for every LED. The graph indicates that $50 \%$ of the observers may not notice the colour change if the lumen depreciation is within a $5 \%$ of each used LED from the rated luminous flux of the individual LED. However, Figure 7 also implies that the colour change will be significantly noticeable from $7.5 \%$ of lumen depreciation of each used LED from the rated luminous flux of the individual LED at $5500 \mathrm{~K}$.

The application note of MAZet ${ }^{31}$ used the true colour sensor to monitor the LED colour for multi-coloured LED lighting systems which could be a closer approach to our work. They found, at the neutral CCT $(4500 \mathrm{~K})$, the optimization can improve to a $\Delta u^{\prime} v^{\prime}$ $=0.0021$. In our lighting system, the simulation shows that the improvement of a $\Delta u^{\prime} v^{\prime}$ is to 0.0018 within the $95 \%$ coverage range at $4500 \mathrm{~K}$.

\section{Conclusions}

Although multi-coloured LED lighting systems are popular nowadays, they often suffer from poor colour stability and reproducibility from system-to-system due to colour and luminous flux variation. We propose a methodology for stabilizing a five-coloured LED lighting system by introducing a colour control algorithm inside the system, covering a wide white gamut to achieve chromaticity and luminous flux stability during operation. 
The LED lighting system produces a tunable white light output; with CCTs ranging from $3000 \mathrm{~K}$ to $6000 \mathrm{~K}$ with a luminous flux dimming capability for each CCT setting. The implemented colour control is a combination of a pre-calibrated lookup table for CCT and $\Phi_{V}$ in combination with a calibrated tristimulus colour sensor for luminous flux and chromaticity control. Additional $D_{u v}$ correction is needed and implemented to bring the output variations near the just noticeable limit.

The colour control system performance is analyzed by using a Monte Carlo simulation process. This process simulates the SPD of the mixed light output based on stochastic variations in the input parameters: LED flux and wavelength bin, junction temperature, driving current, ambient temperature, driver electronics, and colour sensor spectral response. For the stimulated SPD variations, and hence variations in CCT, $D_{u v}$ and $\Phi_{V}$ (as the outputs of the Monte Carlo process) the colour sensor provides real-time feedback of the light output. The pre-calibrated lookup table is made by spectral optimization of SPD over a range of CCT values and luminous flux values covering the operational range for a particular LED lighting system. It is based on the spectral measurements of five-coloured LED channels and the optimized settings of low $D_{u v}\left( \pm 2 \cdot 10^{-3}\right)$, a general colour rendering index (CRI) of $R_{a}>85$, and a particular CRI $R_{9}>40$.

The control system has been shown to be able to regulate the light output and chromaticity. At $5500 \mathrm{~K}, D_{u v}$ variations are reduced from approximately 0.025 to 0.001 . 
The controlled stabilizing system decreases the average uncertainty of luminous flux from $8 \%$ to $2.5 \%$ and similarly reduces the corresponding chromaticity from the uncontrolled system average 0.0193 to the controlled system average 0.00125 in $\Delta u^{\prime} v^{\prime}$ by using a $95 \%$ coverage interval. We have thus shown that with our methodology, similar but varying lighting systems, utilizing other LED bins and environmental conditions, can be controlled to within chromaticity variations corresponding to twice the JND, without doing any additional calibrations.

\section{Funding}

This research was made possible through the project "V8 Light engine - a revolution for coloured light" funded by The Danish National Advanced Technology Foundation (037-2011-3) and the innovation consortium "Center for LED metrology".

\section{Acknowledgements}

We would also like to thank Maria Louisa Rosenberg Welling from the Technical University of Denmark (DTU) Fotonik for her editorial help. 


\section{References}

1. Kim JK, Schubert EF. Transcending the replacement paradigm of solid-state lighting. Optics Express 2008; 16(26): 21835-21842.

2. De Almeida A, Santos B, Paolo B, Quicheron M. Solid state lighting review potential and challenges in Europe. Renewable and Sustainable Energy Reviews 2014; 34: 30-48.

3. US Department of Energy. LED Basics. 2016. Retrieved from: http://energy.gov/eere/ssl/led-basics

4. Oh JH, Yang SJ, Sung Y-G, Do YR. Excellent color rendering indexes of multipackage white LEDs. Optics Express 2012; 20(18): 20276.

5. Lei J, Gu X, Liu M. Spectral assemblage using LEDs to obtain specified characteristics. Applied Optics 2014; 53(35): 8151-8156.

6. Zhang M, Chen Y, He G. Color temperature tunable white-light LED cluster with extra high color rendering index. Scientific World Journal 2014; 9: 897960. 
7. Dong J, Pandharipande A, Van Driel W, Zhang G. Diagnosing lumen depreciation in LED lighting systems: An estimation approach. IEEE Transactions on Signal Processing 2012; 60(7): 3796-3808.

8. Chhajed S, Xi Y, Li YL, Gessmann T, Schubert EF. Influence of junction temperature on chromaticity and color-rendering properties of trichromatic white-light sources based on light-emitting diodes. Journal of Applied Physics 2005; 97: 1-8.

9. OSRAM. Reliability and Lifetime of LEDs. 2013. Retrieved from: http://www.osram-os.com/Graphics/XPic6/00102625_0.pdf

10. Nimz T, Hailer F, Jensen K. Sensors and feedback control of multi-colour LED systems. LED Professional Review 2012; 32: 46-49.

11. Robinson S, Ashdown I. Polychromatic optical feedback control, stability, and dimming. In: Proceedings of the SPIE, 2006: pp. 633714-633714-10.

12. Man K, Ashdown I. Accurate colorimetric feedback for RGB LED clusters. In: 
Proceedings of the SPIE, 2006: pp. 633702-1.

13. Sisto MM, Gauvin J. Accurate chromatic control and color rendering optimization in LED lighting systems using junction temperature feedback. In: Proceedings of the SPIE, 2014: pp. 919002.

14. He G, Tang J. Spectral optimization of color temperature tunable white LEDs with excellent color rendering and luminous efficacy. Optics Letters 2014; 39(19): $5570-5573$.

15. Li H, Mao X, Han Y, Luo Y. Wavelength dependence of colorimetric properties of lighting sources based on multi-color LEDs. Optics Express 2013; 21(3): 37753783.

16. Liu Z, Liu S, Wang K, Luo X. Optical analysis of color distribution in white LEDs with various packaging methods. IEEE Photonics Technology Letters 2008; 20(24): 2027-2029.

17. MAZeT GmbH. True Color Management for Lighting Systems, 2007. Retrieved from: http://www.energie-und- 
technik.de/fileadmin/media/whitepaper/files/app07226e.pdf

18. Žukauskas A, Vaicekauskas R, Ivanauskas F, Gaska R, Shur MS. Optimization of white polychromatic semiconductor lamps. Applied Physics Letters 2002; 80(2): 234-236.

19. Ries H, Leike I, Muschaweck J. Optimized additive mixing of colored lightemitting diode sources. Optical Engineering 2004; 43(7): 1531.

20. Muthu S, Chang C, Bruning G. White LED luminary light control system. US 6,441,558 B1, 2002.

21. Aldrich $\mathrm{M}$, Zhao N, Paradiso J. Energy efficient control of polychromatic solid state lighting using a sensor network. Tenth International Conference on Solid State Light, Proceedings of the SPIE 2010; 7784(1): 778408.

22. Ackermann B, Schulz V, Martiny C, Hilgers A, Zhu X. Control of LEDs. IEEE Industrial Applications Society 2006; 5: 2608-2615.

23. Commission Internationale de l'Eclairage. CIE TN 001:2014 Chromaticity 
Difference Specification for Light Sources. Vienna: CIE, 2014.

24. Chakrabarti M, Pedersen HC, Petersen PM, Poulsen C, Poulsen PB, Dam-Hansen C. High-flux focusable color-tunable and efficient white-light-emitting diode light engine for stage lighting. Optical Engineering 2016; 55(8): doi 10.1117/1.OE.55.8.085101.

25. Chakrabarti M, Thorseth A, Corell DD, Dam-Hansen C. A white-cyan-red LED system for low correlated colour temperature lighting. Lighting Research and Technolnology. First published 3 August 2015, doi 1477153515608416.

26. Chakrabarti M, Thorseth A, Jepsen J, Dam-Hansen C. Monte carlo analysis of multicolour LED light engine. In: Proceedings of the $28^{\text {th }}$ CIE Session, Manchester, UK. 28 June - 4 July 2015. Vienna: CIE: p. OP60 526.

27. Gamma Scientific. GS-1150 Handheld Spectrophotometers. 2015. Retrieved from: http://www.elliotscientific.com/image/data/pdf/Gamma/GS1150_Datasheet_949.pdf

28. Commission Internationale de l'Eclairage. Method of Measuring and Specifying 
Colour Rendering Properties of Light Sources. CIE Publication 13.3. Vienna, CIE, 1995.

29. Rea MS, Freyssinier JP. White lighting: A provisional model for predicting perceived tint in "white" illumination. Color Research and Application 2014; 39: 466-479.

30. Ohno Y, Fein M. Vision experiment on acceptable and preferred white light chromaticity for lighting. In: CIE 2014 Lighting Quality and Energy Efficiency, CIE Publication x039:2014. Vienna: CIE, pp. 192-199.

31. MAZeT GmbH. Accuracy Optimization of True Color Sensor Solutions for LED Color Control of Multiple LED Light Sources. 2012. Retrieved from: http://www.mazet.de/en/downloads/product-customer-information/whitepaper.html 


\section{Figure captions}

Figure 1. Schematic diagram of the colour control system of the multi-coloured tunable (3000 K to $6000 \mathrm{~K}$ ) white LED lighting system

Figure 2. Resultant SPDs as the target (black line) in a five colour mixing LED system at two different target CCTs ( $3000 \mathrm{~K}$ and $5800 \mathrm{~K}$ in (a) - (b)) and the corresponding reference SPDs (purple line). Individual SPDs of the five coloured LEDs are also shown by different colours.

Figure 3. (a) - (d) represent the probability distributions within $95 \%$ coverage interval (green) of uncontrolled CCT, $D_{u v}, \mathrm{CRI} R_{a}$, and $R_{g}$, respectively, for 2000 iterations while the red colour is indicating the same for outside the $95 \%$ coverage interval for a target CCT of $5500 \mathrm{~K}$ with $\Phi_{V}=8000 \mathrm{Im}$.

Figure 4. (a) - (d) represent the probability distributions within $95 \%$ coverage interval (green) of controlled CCT, $D_{u v}, \mathrm{CRI} R_{a}$, and $R_{g}$, respectively, for 2000 iterations. The red colour is indicating the same for outside the 95\% coverage interval at a target CCT of $5500 \mathrm{~K}$ with $\Phi_{V}=8000 \mathrm{Im}$

Figure 5. The example of uncontrolled and controlled solutions for the lighting system at $5500 \mathrm{~K}$ in the $\mathrm{CIE} 1976\left(u^{\prime}-v^{\prime}\right)$ diagram

Figure 6. Controlled light output from the lighting system while the CCT is tuned from $3000 \mathrm{~K}$ to $6000 \mathrm{~K}$ with $100 \mathrm{~K}$ resolution at $8000 \mathrm{Im}$

Figure 7. Simulated change in $\Delta u^{\prime} v^{\prime}$ for a target CCT of $3500 \mathrm{~K}, 4500 \mathrm{~K}$, and $5500 \mathrm{~K}$ 
according to lumen depreciation in percent, for every LED of the lighting system.

Table 1. CCT, $D_{u v}$, and $\Phi_{V}$ variations within $95 \%$ coverage interval at target CCT settings with $\Phi_{V}=8000 \mathrm{~lm}$

\begin{tabular}{ccccccc}
\hline $\begin{array}{c}\text { Target } \\
\text { CCT [K] }\end{array}$ & $\begin{array}{c}\Delta \mathrm{CCT}[\mathrm{K}] \\
\text { (uncontrolled) }\end{array}$ & $\begin{array}{c}\Delta \mathrm{CCT}[\mathrm{K}] \\
\text { (controlled) }\end{array}$ & $\begin{array}{c}\Delta D_{u v} \\
\text { (uncontrolled) }\end{array}$ & $\begin{array}{c}\Delta D_{u v} \\
\text { (controlled) }\end{array}$ & $\begin{array}{c}\Delta \Phi_{V}[\mathrm{Im}] \\
\text { (uncontrolled) }\end{array}$ & $\begin{array}{c}\Delta \Phi_{V}[\mathrm{~lm}] \\
\text { (controlled) }\end{array}$ \\
\hline 3500 & 1070 & 43 & 0.0251 & 0.0010 & 785 & 209 \\
4000 & 732 & 60 & 0.0190 & 0.0008 & 637 & 199 \\
4500 & 742 & 71 & 0.0160 & 0.0013 & 610 & 190 \\
5000 & 722 & 112 & 0.0189 & 0.0022 & 598 & 202 \\
5500 & 940 & 133 & 0.0252 & 0.0011 & 704 & 209 \\
6000 & 849 & 150 & 0.0227 & 0.0005 & 663 & 208 \\
\hline
\end{tabular}


Table 2. CCT, $D_{u v}$, and $\Phi_{V}$ variations within $95 \%$ coverage interval at a target CCT $=3000$ $\mathrm{K}$ with $\Phi_{V}=4000 \mathrm{Im}$ and $8000 \mathrm{Im}$, respectively

\begin{tabular}{cccccccc}
\hline Target & Target & $\Delta \mathrm{CCT}[\mathrm{K}]$ & $\Delta \mathrm{CCT}[\mathrm{K}]$ & $\Delta D_{u v}$ & $\Delta D_{u v}$ & $\Delta \Phi_{V}[\mathrm{Im}]$ & $\Delta \Phi_{V}[\mathrm{Im}]$ \\
CCT $[\mathrm{K}]$ & $\Phi_{V}[\mathrm{~lm}]$ & (uncontrolled) & (controlled) & (uncontrolled) & (controlled) & (uncontrolled) & (controlled) \\
\hline 3000 & 4000 & 361 & 8 & 0.0067 & $3.8 \times 10^{-5}$ & 257 & 88 \\
& 8000 & 1620 & 6 & 0.0365 & $1.2 \times 10^{-4}$ & 874 & 380 \\
\hline
\end{tabular}


Table 3. Luminous flux depreciation and LED failures among 210 colour LEDs

\begin{tabular}{cccc}
\hline Type of LEDs & $\begin{array}{c}\text { Total numbers in } \\
\text { lighting system }\end{array}$ & $\begin{array}{c}\text { Acceptable Luminous flux } \\
\text { depreciation [\%] }\end{array}$ & $\begin{array}{c}\text { Acceptable number of LED } \\
\text { failures }\end{array}$ \\
\hline Royal blue & 18 & 15.2 & 3 \\
Blue & 24 & 7.6 & 2 \\
Red & 12 & 4 & 0 \\
Green & 30 & 2.3 & 0 \\
White & 126 & 2.3 & 3 \\
\hline
\end{tabular}


